\title{
Effects of thrombin on the secondary cerebral injury of perihematomal tissues of rats after intracerebral hemorrhage
}

\author{
C.M. Liu ${ }^{1}$, B.Z. Shi ${ }^{2}$ and J.S. Zhou ${ }^{1}$ \\ ${ }^{1}$ Department of Neurology, Nanjing First Hospital, \\ Nanjing Medical University, Nanjing, China \\ ${ }^{2}$ Yuhua Hospital of Yuhuatai District, Nanjing City, China \\ Corresponding author: J.S. Zhou \\ E-mail: baozhushicn@126.com
}

Genet. Mol. Res. 13 (2): 4617-4626 (2014)

Received June 12, 2013

Accepted October 3, 2013

Published June 18, 2014

DOI http://dx.doi.org/10.4238/2014.June.18.4

\begin{abstract}
This study aimed to investigate the effects of thrombin released in hematoma after intracerebral hemorrhage (ICH) on the cerebral injury of perihematomal tissues and to evaluate the protection effect of hirudin on the cerebral injury after ICH. We used the autologous uncoagulated blood injection method to prepare the ICH rat model, and all rats were randomly divided into a normal group, an ICH group, or a hirudin group. At different time points, rat heads were cut to harvest brain sections. Immunohistochemical staining, histochemical staining, and hematoxylin and eosin staining were conducted for CD34, microglia, and neutrocytes. CD34-positive microvessels were most abundant in brain tissues of the sham-operation group. At $12 \mathrm{~h}$ after $\mathrm{ICH}, \mathrm{CD} 34$ expression reduced and reached the minimum level at $72 \mathrm{~h}(\mathrm{P}<0.01)$. At $6 \mathrm{~h}$ after $\mathrm{ICH}$, microglia expression was visible and reached a peak at $48 \mathrm{~h}(\mathrm{P}<0.01)$. At $12 \mathrm{~h}$ after $\mathrm{ICH}$, neutrocyte infiltration was visible and the number was greatest at $48 \mathrm{~h}(\mathrm{P}<0.01)$. The early application of hirudin after ICH could significantly reduce microglia and neutrocyte expression and could significantly slow down
\end{abstract}


the CD34 decrease trend $(\mathrm{P}<0.01)$. However, hirudin application in the edematization stage after ICH did not significantly increase CD34positive microvessel abundance $(\mathrm{P}>0.05)$. A thrombin-mediated inflammatory reaction is involved in the cerebral injury after $\mathrm{ICH}$, and the early application of hirudin has a protective effect.

Key words: Intracerebral hemorrhage; Rats; Thrombin; Hirudin

\section{INTRODUCTION}

Many studies have shown that in the pathophysiological process of secondary cerebral injury and encephaledema after intracerebral hemorrhage (ICH), thrombin is a very important toxic substance. In addition, thrombin is a multi-functional serum serine protease generated from inactive prothrombin that can catalyze fibrinogen to convert into fibrin. Therefore, thrombin plays a key role in the coagulation cascade reaction (Fujimoto et al., 2007; Ten Cate et al., 2012). In the last decade, thrombin has been established as one of the most important extracellular signaling molecules, and participates in a series of pathophysiological processes such as ischemic, hemorrhagic, and traumatic cerebral injuries caused by activation of the thrombin receptor (Xi et al., 2006). Lee et al. (1997) studied the relationship of thrombin with encephaledema and found that encephaledema in early ICH (within $24 \mathrm{~h}$ ) was cytotoxic encephaledema caused by the toxic action of thrombin, while encephaledema in later ICH (after $24 \mathrm{~h}$ ) was vasogenic encephaledema caused by blood-brain barrier (BBB) damage. CD34 is a single-chain transmembrane protein with a molecular weight of $105-120 \mathrm{kDa}$, and is one of the main indicators of a vasogenic lesion (Furness and McNagny, 2006; Gangenahalli et al., 2006). In tumor tissues, CD34 expression increases due to vascular proliferation. In hemorrhage and edema, CD34 expression is reduced due to vascular endothelial damage. Therefore, it is feasible to indirectly assess BBB permeability by detecting CD34 levels in perihematomal brain tissue (Mackenzie and Clayton, 1999).

Previous studies (Sharp et al., 2008; Wu et al., 2010) suggest that thrombin can cause a secondary injury by recruiting inflammatory cells to form an excessive inflammatory reaction. In the early inflammatory reaction, there are two major cellular components: the peripheral blood-derived exogenous neutrophilic granulocytes and neutrophils and the central nervous tissue-derived endogenous microglia.

Microglia are effector cells in inflammation and immune reactions in the central nervous system. Normally, microglia are in a resting state and are few in number (Moller et al., 2000). RCA-1 is a type of glycoprotein composed of two $60-\mathrm{kDa}$ subunits. It specifically combines with $\beta$-D galactosyl on the surface of activated microglia using a mode similar to the antigen-antibody reaction. In the resting state, $\beta$-D galactosyl does not combine with RCA-1 (Xue and Del Bigio, 2001). Ruan et al. (2008) suggested that activated microglia could release neurotoxic substances and inflammatory factors to cause inflammatory injury to brain tissue. Kawai et al. (2001) showed that the directional injection of thrombin into the rat brain could generate apparent encephaledema, cause inflammation, and induce apoptosis.

Thrombin-mediated overactivation of microglia and leukocytes can cause pathological damage to the central nervous system, and it is a potential therapeutic target for reducing tissue damage (Hu et al., 2011; Babu et al., 2012). Hirudin is a known, potent, specific, and exogenous inhibitor of thrombin. The coagulation of $50 \mu \mathrm{L}$ whole blood generates about 8-10 
$\mathrm{U}$ thrombin, and $1 \mathrm{U}$ hirudin can inhibit $1 \mathrm{U}$ thrombin (Lee et al., 1996). Therefore, $10 \mathrm{U}$ hirudin can inhibit the activity of thrombin in $50 \mu \mathrm{L}$ whole blood.

When active thrombin diffuses into the surrounding brain parenchyma after formation of ICH and initiates the blood coagulation chain, its toxic effects on the central nervous system are dangerous. Thrombin causes secondary cerebral injury and encephaledema by binding the thrombin receptor in the brain (Nakamura et al., 2005; Ohnishi et al., 2007). We modeled ICH by injecting autologous uncoagulated blood into the rat caudate nucleus using the stereotactic technique. We observed the expression of microglia and leukocyte markers that were mediated by the upstream inflammatory signaling molecule, thrombin. We characterized the extent of BBB damage and conducted an intervention treatment with its specific inhibitor, hirudin, to explore the role of thrombin-mediated inflammatory reactions in cerebral injury that occur after ICH.

\section{MATERIAL AND METHODS}

\section{Animals}

Healthy male Sprague-Dawley (SD) rats weighing from 250 to $320 \mathrm{~g}$ were provided by the Experimental Animal Center of Nanjing Medical University (strain number: 97001). The SD rats were divided into three groups: the sham-operation group $(\mathrm{N}=6)$, the conventional ICH group, and the intervention group. In the conventional ICH group, the rats were divided into 7 subgroups according to different time points after ICH: $6,12,24,48,72 \mathrm{~h}, 1$ week, and 2 weeks $(\mathrm{N}=6$ per group). In addition, the intervention group was divided into 4 subgroups $(\mathrm{N}=6$ per group), in which hirudin intervention was conducted in two subgroups, and normal saline intervention was conducted in two subgroups. We defined success in this model when rat brain sections showed the apparent presence of round-, oval-, or irregular-shaped hematomas, no needle tract reflux of blood, no subarachnoid hemorrhage, and no hemorrhage rupturing into ventricles. Furthermore, rats that were unconscious or died after the operation were excluded. According to our success criteria, 72 rats showed successful outcomes. We bred experimental animals and used disease protocols in this study that strictly complied with the National Standards of Laboratory Animal Use and that were approved by the Experimental Animal Management Committee of Nanjing Medical University.

\section{ICH model preparation}

The rat ICH model was prepared according to the method of Xue and Del Bigio (2000). Rats were anesthetized with $300 \mathrm{mg} / \mathrm{kg} 10 \%$ chloral hydrate by intraperitoneal injection and fixed onto a stereotaxic apparatus. An approximately $10-\mathrm{mm}$ incision was made along the scalp midline to open the periosteum and expose the anterior fontanelle. A microsyringe was positioned $0.5 \mathrm{~mm}$ in front of the anterior fontanelle. At $3 \mathrm{~mm}$ from the left side of the midline, a $0.5-\mathrm{mm}$ small hole was drilled with a micro-hand drill. Subsequently, the tail was shorn to draw blood, and heparin was added for anticoagulation. Next, $50 \mu \mathrm{L}$ autologous uncoagulated blood was taken with the microsyringe. Along the drilled small hole, the microsyringe needle was inserted to a 6-mm depth (to reach the caudate nucleus), and the blood was injected at a constant rate of $20 \mu \mathrm{L}$ per min. After the blood was injected, the needle was held in place for 5 min before it was removed. Finally, the incision was sutured. In the sham-operation group, the early steps of the operation were the same, but the blood was not injected. After $2 \mathrm{~h}$, rats were 
decapitated and brains were harvested. In the experimental intervention group, $15 \mu \mathrm{L}$ normal saline containing $10 \mathrm{U}$ hirudin ( $15 \mu \mathrm{L}$ normal saline was only used in the control group) was injected into the original hematoma location at $5 \mathrm{~min}$ and $24 \mathrm{~h}$ after ICH. Rats were decapitated and the brains were harvested at $24 \mathrm{~h}$ after the intervention in each subgroup.

\section{Preparation of specimens and tissue sectioning}

For various groups of rats, excessive chloral hydrate was injected to maintain deep anesthesia at different time points after hemorrhage. The thoracic cavities were rapidly opened, and lavage was conducted with normal saline via the heart. After decapitating rats, the brains were harvested and placed into neutral formalin for fixation. Specimens were hydrated, embedded with paraffin wax, and sliced continuously into 5 - $\mu \mathrm{m}$ thick sections with a slicer. Consecutive sections were used for conventional immunohistochemical, hematoxylin and eosin (HE), and RCA-1-agglutinin-labeled immunohistochemical staining.

\section{Immunohistochemical staining}

Sections were dewaxed and placed into water. After $3 \% \mathrm{H}_{2} \mathrm{O}_{2}$ was added, the sections were incubated for $10 \mathrm{~min}$ at room temperature. After antigen retrieval liquid (the antigen was repaired by microwave) was added, the sections were incubated for $30 \mathrm{~min}$ at room temperature. Subsequently, goat serum blocking solution was added drop-wise, and the sections were incubated for $30 \mathrm{~min}$ at room temperature. Next, primary antibody (rabbit anti-rat CD34 monoclonal antibody) was added, and the sections were incubated at $4{ }^{\circ} \mathrm{C}$ overnight. Biotinylated secondary antibody (goat anti-rabbit IgG) was added, and the sections were incubated for $30 \mathrm{~min}$ at room temperature. Afterward, SABC reagent was added drop-wise and the sections were incubated for $20 \mathrm{~min}$ at room temperature. Subsequently, 3,3'-diaminobenzidine (DAB) developing was conducted by adding fresh DAB solution, and color density was observed under a microscope. The sections were restained with hematoxylin, hyalinized, and mounted.

Sections were dewaxed and placed into water, and $0.01 \%$ tryptase was used to digest for 20 to $45 \mathrm{~min}$ to expose the antigen. Subsequently, the sections were washed twice ( $5 \mathrm{~min}$ per wash), incubated in bovine serum albumin for $5 \mathrm{~min}$, incubated in the mixture of biotinylated RCA-1 agglutinin and phosphate-buffered saline $(1: 1000)$ at $4{ }^{\circ} \mathrm{C}$ for $48 \mathrm{~h}$, incubated in 1:100 ABC reagent for $60 \mathrm{~min}$, and then washed with Tris-buffered saline twice $(5 \mathrm{~min}$ per wash). Subsequently, development by DAB was conducted and the sections were restained with hematoxylin, hyalinized, and mounted.

\section{Immunohistochemical analyses}

Immunohistochemical and histochemical staining of CD34 and microglia occurred in the cytoplasm and on the cell membrane, appearing as brown granules. Under high-magnification microscopy (400X), 4 different visual fields near the hematoma were randomly selected, and numbers of CD34-positive microvessels, positively stained microglia, and infiltrating leukocytes were counted for each high-magnification visual field. Data are reported as means \pm standard deviation and analyzed using the computerized pathological image management system 4.10 (Image Processing Center of Beihang University). 


\section{Statistical analysis}

One-way analysis of variance (ANOVA) was used for statistical processing, and the Q-test was used for comparisons between mean values of multiple samples. Data were analyzed using the SPSS15.0 software package. $\mathrm{P}<0.05$ was considered to represent a statistically significant difference.

\section{RESULTS}

\section{Patterns of CD34 expression, microglia activation, and leukocyte infiltration after ICH}

In the sham-operation group, no leukocyte infiltration or microglia staining were visible in the brain tissue (Figure 1A), whereas CD34-positive microvessels were abundant (Figure 1B). At $12 \mathrm{~h}$ after $\mathrm{ICH}, \mathrm{CD} 34$ staining became somewhat reduced and reached a minimum level by $72 \mathrm{~h}$ (Figure 1C). By 7 days, the CD34 staining intensity was eventually restored to normal levels. At $6 \mathrm{~h}$ after ICH, RCA-1 agglutinin-labeled brown microglia were visible around the hematoma and reached the peak at $48 \mathrm{~h}$ (Figure 2A), and a small amount of expression persisted for 2 weeks. At $12 \mathrm{~h}$ after ICH, leukocyte infiltration was visible in and around hematoma, reaching a peak at $48 \mathrm{~h}$ (Figure 2B), and then gradually decreasing and disappearing by 2 weeks. There were significant differences observed between various experimental groups and between the experimental and sham-operation groups $(\mathrm{P}<0.01)$ (Table 1$)$.

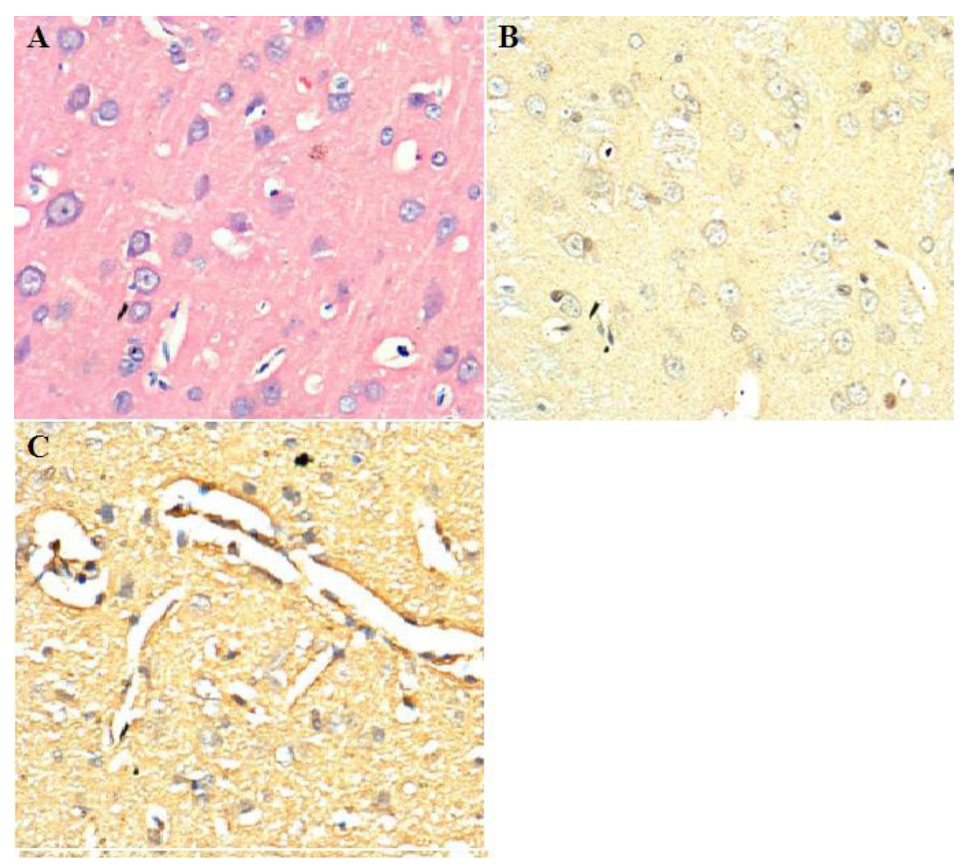

Figure 1. A. No neutrocyte expression in the sham-operation group (HE, 400X). B. No microglia expression in the sham-operation group (RCA-1, 400X). C. CD34 immunoreactive-positive microvessel number of the shamoperation group (S-P immunohistochemistry 400X; SP Kit, Histostain ${ }^{\mathrm{TM}}$-Plus Kits). 


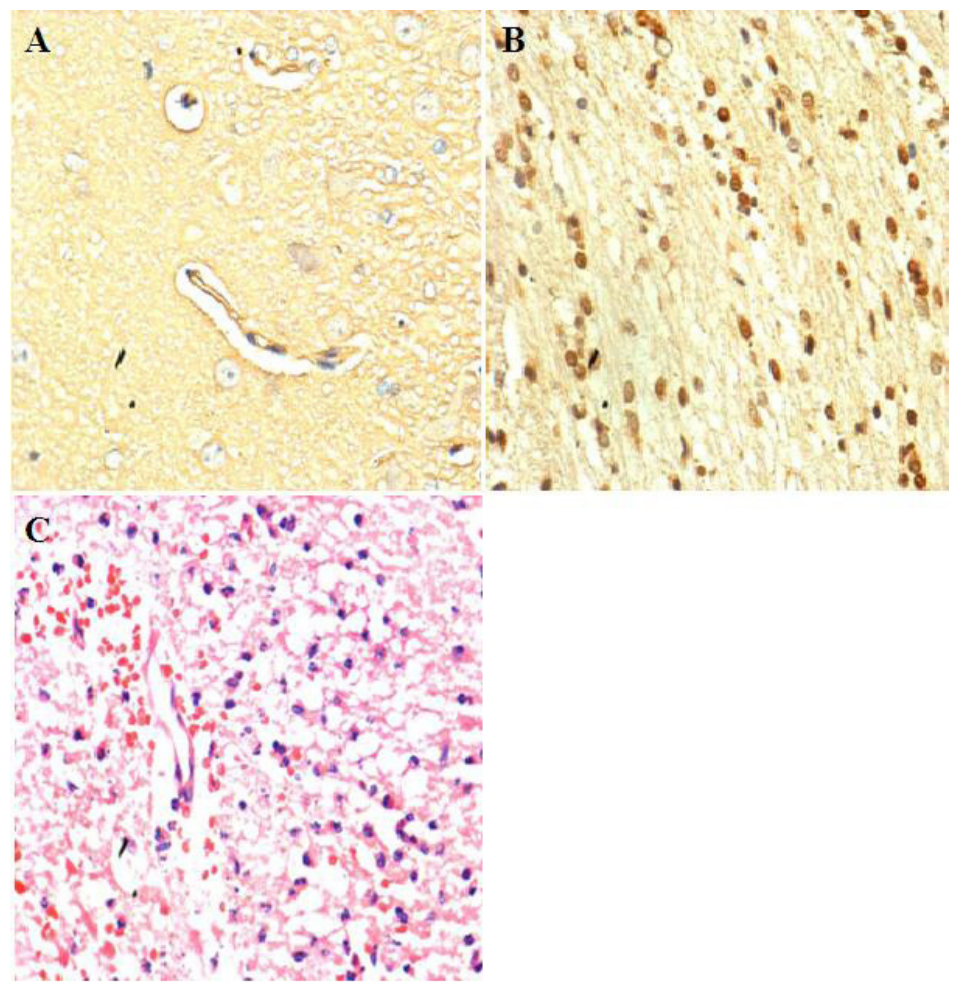

Figure 2. A. Perihematomal CD34 immunoreactive-positive microvessel number at $72 \mathrm{~h}$ after ICH (S-P immunohistochemistry 400X; SP Kit, Histostain ${ }^{\mathrm{TM}}$-Plus Kits). B. Microglia expression at $48 \mathrm{~h}$ after ICH (RCA-1, $400 \mathrm{X})$. C. Neutrocyte infiltration at $48 \mathrm{~h}$ after ICH (HE, 400X).

\begin{tabular}{|c|c|c|c|c|}
\hline \multirow[t]{2}{*}{ Group } & \multirow[t]{2}{*}{ Case } & \multicolumn{3}{|c|}{ Positive cell or microvessel count } \\
\hline & & White blood cell & Microglia & CD34 \\
\hline Sham-operation group & 6 & $0.00 \pm 0.00$ & $0.00 \pm 0.00$ & $34.66 \pm 1.21$ \\
\hline ICH $6 \mathrm{~h}$ & 6 & $0.00 \pm 0.00$ & $9.66 \pm 1.21 *$ & $33.73 \pm 3.60^{\#}$ \\
\hline ICH $12 \mathrm{~h}$ & 6 & $6.16 \pm 0.75 *$ & $18.67 \pm 1.86^{*}$ & $24.82 \pm 2.92^{*}$ \\
\hline ICH $24 \mathrm{~h}$ & 6 & $13.33 \pm 1.75^{*}$ & $33.83 \pm 4.66^{*}$ & $19.00 \pm 1.41 *$ \\
\hline ICH $48 \mathrm{~h}$ & 6 & $31.66 \pm 3.26^{*}$ & $64.16 \pm 6.64 *$ & $13.67 \pm 1.21^{*}$ \\
\hline $\mathrm{ICH} 72 \mathrm{~h}$ & 6 & $15.33 \pm 2.16^{*}$ & $31.66 \pm 3.14 *$ & $5.16 \pm 0.75^{*}$ \\
\hline ICH 1 week & 6 & $5.00 \pm 0.89 *$ & $11.33 \pm 2.16^{*}$ & $32.50 \pm 2.43^{\#}$ \\
\hline ICH 2 weeks & 6 & $0.00 \pm 0.00$ & $6.67 \pm 0.82 *$ & $33.34 \pm 1.21^{\#}$ \\
\hline
\end{tabular}

Data are reported as means $\pm \mathrm{SD} .{ }^{*} \mathrm{P}<0.01,{ }^{\sharp} \mathrm{P}>0.05$ versus the sham-operation group.

\section{Comparisons of inflammatory indicators between the 5-min and 24-h ICH intervention groups}

At 5 min after ICH, $15 \mu \mathrm{L}$ normal saline or $15 \mu \mathrm{L}$ normal saline $+10 \mathrm{U}$ hirudin were injected at the original hematoma location. After $24 \mathrm{~h}$, rats were decapitated and 
brains were harvested in order to compare the intervention group at $5 \mathrm{~min}$ after $\mathrm{ICH}$ to the intervention group at $24 \mathrm{~h}$ after ICH. In the hirudin intervention group, perihematomal microglia and leukocyte abundance were significantly reduced, and changes in CD34 expression levels were less affected $(\mathrm{P}<0.01)$ (Figures $2 \mathrm{C}, 3 \mathrm{~A}$ and $\mathrm{B})$. However, normal saline had no such effect $(\mathrm{P}>0.05$; Table 2$)$.

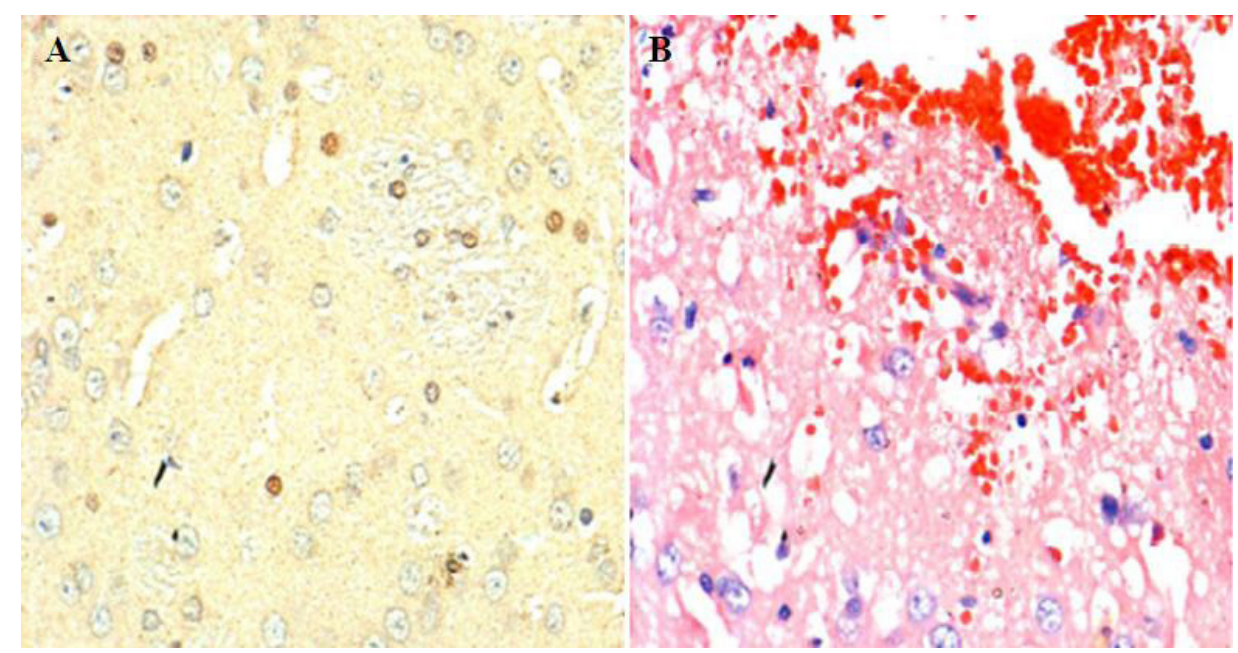

Figuer 3. A. Microglia expression after hirudin intervention at $5 \mathrm{~min}$ after ICH (RCA-1, 400X). B. Neutrocyte infiltration after hirudin intervention at $5 \mathrm{~min}$ after ICH (HE, 400X).

Table 2. Comparison of the positive number of white blood cell, microglia and the number of positive microvascular CD34 between different groups.

\begin{tabular}{lcccc}
\hline Group & Case & \multicolumn{3}{c}{ Positive cell } \\
\cline { 3 - 4 } & & White blood cell & Microglia & CD34 \\
\hline $24-\mathrm{h} \mathrm{ICH}$ & 6 & $13.33 \pm 1.75$ & $33.83 \pm 4.66$ & $19.00 \pm 1.41$ \\
$15 \mu \mathrm{L}$ normal saline & 6 & $12.66 \pm 2.06^{*}$ & $32.16 \pm 2.13^{\#}$ & $2.56 \pm 1.87^{*}$ \\
$15 \mu \mathrm{L}$ normal saline + hirudin & 6 & $5.50 \pm 1.87^{*}$ & $17.33 \pm 2.16^{*}$ & $32.16 \pm 2.31^{*}$ \\
\hline
\end{tabular}

Data are reported as means $\pm \mathrm{SD} . * \mathrm{P}<0.01$ for the $24-\mathrm{h} \mathrm{ICH}$ group $v s$ normal saline group . ${ }^{*} \mathrm{P}>0.05$ vs $24-\mathrm{h} \mathrm{ICH}$ group. $\mathrm{ICH}=$ intracelebral hemorrhage.

\section{Effect of hirudin on edematization between the 24- and 48-h ICH intervention groups}

At $24 \mathrm{~h}$ after ICH, $15 \mu \mathrm{L}$ normal saline or $15 \mu \mathrm{L}$ normal saline $+10 \mathrm{U}$ hirudin were injected into the original hematoma location. After $24 \mathrm{~h}$, rats were decapitated and their brains were harvested in order to compare the intervention group at $5 \mathrm{~min}$ after ICH to the intervention group at $24 \mathrm{~h}$ after ICH. In the hirudin intervention group, perihematomal microglia and leukocyte abundance remained significantly inhibited $(\mathrm{P}<0.05)$ (Figure $4 \mathrm{~A})$, and the CD34-positive microvessel number did not significantly increase $(\mathrm{P}>0.05)$ (Figure 4B and Table 3). 


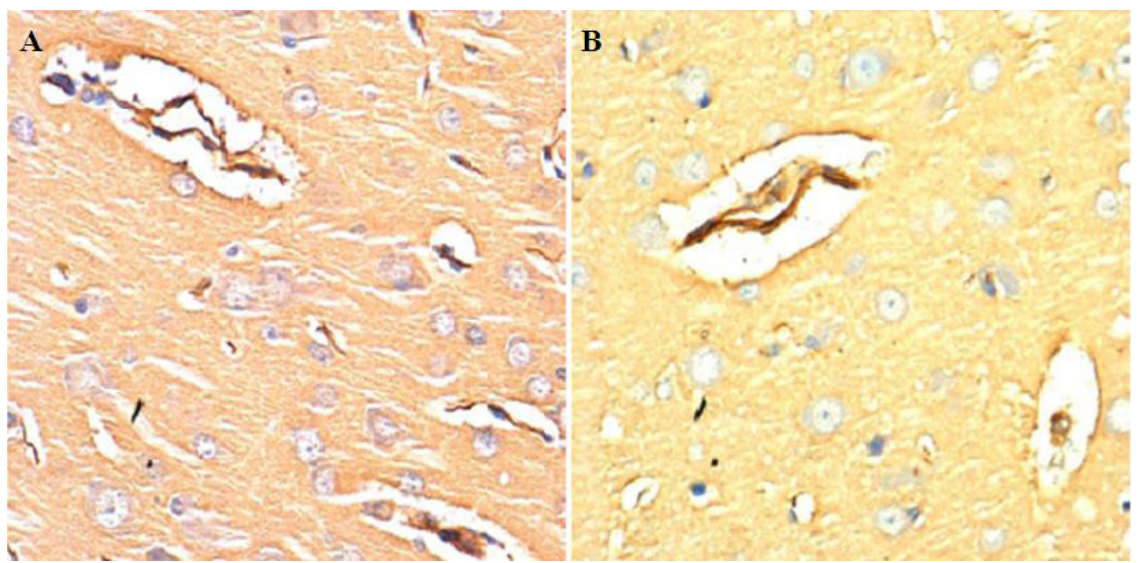

Figure 4. A. CD34 microvessel number after hirudin intervention at $5 \mathrm{~min}$ after ICH (SP, 400X). B. CD34 microvessel number after hirudin intervention at $24 \mathrm{~h}$ after ICH (S-P immunohistochemistry 400X; SP Kit, Histostain $^{\mathrm{TM}}$-Plus Kits).

Table 3. Comparison of the positive number of white blood cell, microglia and the number of positive microvascular CD34 between the intracerebral hemorrhage (ICH) intervention group for $24 \mathrm{~h}$ and ICH for $48 \mathrm{~h}$.

\begin{tabular}{|c|c|c|c|c|}
\hline \multirow[t]{2}{*}{ Group } & \multirow[t]{2}{*}{ Case } & \multicolumn{3}{|c|}{ Positive cell } \\
\hline & & White blood cell & Microglia & $\mathrm{CD}_{34}$ \\
\hline 48-h ICH & 6 & $31.66 \pm 3.26$ & $64.16 \pm 6.64$ & $13.67 \pm 1.21$ \\
\hline $15 \mu \mathrm{L}$ normal saline & 6 & $33.50 \pm 4.03^{\#}$ & $66.16 \pm 7.78^{\#}$ & $12.33 \pm 1.36$ \\
\hline $15 \mu \mathrm{L}$ normal saline + hirudin & 6 & $22.66 \pm 3.38^{*}$ & $42.66 \pm 4.76^{*}$ & $13.16 \pm 1.47^{\#}$ \\
\hline
\end{tabular}

Data are reported as means \pm SD. $* \mathrm{P}<0.05$ and ${ }^{*} \mathrm{P}>0.05$ vs the 48 -h ICH group.

\section{DISCUSSION}

Our experimental results showed that CD34 expression in the sham-operation group was the strongest, and that this group had the most microvessels. At $12 \mathrm{~h}$ after ICH, perihematomal CD34 expression began to decline and reached a minimum level at $72 \mathrm{~h}$, suggesting that BBB permeability had reached its peak level. After 7 days, it gradually returned to its normal level, which was in line with the results of Lee et al. (1997), and agreed with the encephaledema peak and critical condition times of clinical ICH patients (2-3 days), indicating that thrombin plays an important role in the pathogenesis of BBB damage.

We did not observe leukocyte infiltration or microglia staining in brain tissue in the sham-operation group. By $12 \mathrm{~h}$ after ICH, perihematomal and internal leukocyte infiltration was more obvious and reached a peak at $48 \mathrm{~h}$, before receding to a minimum (at $72 \mathrm{~h}$ ) prior to the decline in CD34-positive microvessel abundance. This indicated that the inflammatory mediator thrombin can recruit neutrocytes to gather around the hematoma and participate in BBB damage. Recruited leukocytes migrate from the endothelial cell layer by interacting with adhesion molecules on endothelial cells to break through the first mechanical barrier of the BBB (cerebral vascular endothelial cells). Additionally, activated leukocytes can secrete matrix metalloproteinases to activate the plasmin system, and can degrade the extracellular matrix to damage the second mechanical barrier of the BBB (the basement membrane) (Liu et 
al., 2010). BBB damage exposes the base of the brain to intravascular serum proteins, moisture, and harmful substances that exosmose and induce vasogenic encephaledema (Keep et al., 2008). Encephaledema represents the central link of the secondary attachment of neurons after $\mathrm{ICH}$, and is also a main marker of secondary cerebral injury after ICH.

This study showed that no activated microglia were present in the rat brains of the sham-operation group or in the ICH contralateral rat brain, whereas many activated microglia were visible around the hematoma in the ICH group. By $48 \mathrm{~h}$, microglia were most pronounced and this continued until 2 weeks or longer after ICH. In the same model, the peak of BBB damage, namely the time of least CD34 staining, occurred between 48 and $72 \mathrm{~h}$. We found that the timing and distribution of thrombin-induced microglia reactions after ICH occurred prior to BBB damage and that changes of the two were negatively correlated. Moreover, both changes occurred in the area around the hematoma. These patterns suggest that microglia are possibly involved in the process of BBB damage resulting in cerebral injury after ICH.

When we used hirudin to completely deactivate thrombin at 5 min (early) after $\mathrm{ICH}$, microglia activation and leukocyte infiltration were significantly inhibited, while CD34-positive microvessel abundance could be restored to normal levels, indicating that thrombin-induced BBB damage was reversible and early hirudin application could minimize BBB damage. When we applied hirudin $24 \mathrm{~h}$ after ICH (the edematization stage), hirudin could still inhibit microglia activation and leukocyte infiltration, but it did not significantly increase the number of CD34-positive microvessels. These findings suggest that hirudin can inhibit perihematomal inflammatory reactions, but it cannot reverse BBB damage, indicating that the inhibition of hirudin on vasogenic encephaledema has a specific mechanism of action and is most effective when administered quickly. Specifically, the intervention treatment must be conducted in the early stages of activation of these cells and a positive outcome can be achieved only when tissues are in a reversible stage of injury. If the damage exceeds the thresholds of cells, it will cause irreversible cell death. This study provides a theoretical basis for the early application of anti-thrombin drugs to block thrombin-mediated toxicity after ICH in clinical settings.

\section{ACKNOWLEDGMENTS}

Research supported by the Foundation of Science and Technology Development Program, Nanjing Medical University (\#2011NJMU008).

\section{REFERENCES}

Babu R, Bagley JH, Di C, Friedman AH, et al. (2012). Thrombin and hemin as central factors in the mechanisms of intracerebral hemorrhage-induced secondary brain injury and as potential targets for intervention. Neurosurg. Focus 32: E8

Fujimoto S, Katsuki H, Ohnishi M, Takagi M, et al. (2007). Thrombin induces striatal neurotoxicity depending on mitogenactivated protein kinase pathways in vivo. Neuroscience 144: 694-701.

Furness SG and McNagny K (2006). Beyond mere markers: functions for CD34 family of sialomucins in hematopoiesis. Immunol. Res. 34: 13-32.

Gangenahalli GU, Singh VK, Verma YK, Gupta P, et al. (2006). Hematopoietic stem cell antigen CD34: role in adhesion or homing. Stem. Cells Dev. 15: 305-313.

Hu S, Xi G, Jin H, He Y, et al. (2011). Thrombin-induced autophagy: a potential role in intracerebral hemorrhage. Brain Res. 1424: 60-66.

Kawai N, Kawanishi M, Okauchi M and Nagao S (2001). Effects of hypothermia on thrombin-induced brain edema formation. Brain Res. 895: 50-58. 
Keep RF, Xiang J, Ennis SR, Andjelkovic A, et al. (2008). Blood-brain barrier function in intracerebral hemorrhage. Acta Neurochir. Suppl. 105: 73-77.

Lee KR, Colon GP, Betz AL, Keep RF, et al. (1996). Edema from intracerebral hemorrhage: the role of thrombin. $J$. Neurosurg. 84: 91-96.

Lee KR, Kawai N, Kim S, Sagher O, et al. (1997). Mechanisms of edema formation after intracerebral hemorrhage: effects of thrombin on cerebral blood flow, blood-brain barrier permeability, and cell survival in a rat model. J. Neurosurg. 86: $272-278$

Liu DZ, Ander BP, Xu H, Shen Y, et al. (2010). Blood-brain barrier breakdown and repair by Src after thrombin-induced injury. Ann. Neurol. 67: 526-533.

Mackenzie JM and Clayton JA (1999). Early cellular events in the penumbra of human spontaneous intracerebral hemorrhage. J. Stroke Cerebrovasc. Dis. 8: 1-8.

Moller T, Hanisch UK and Ransom BR (2000). Thrombin-induced activation of cultured rodent microglia. J. Neurochem. 75: 1539-1547.

Nakamura T, Xi G, Park JW, Hua Y, et al. (2005). Holo-transferrin and thrombin can interact to cause brain damage. Stroke 36: 348-352.

Ohnishi M, Katsuki H, Fujimoto S, Takagi M, et al. (2007). Involvement of thrombin and mitogen-activated protein kinase pathways in hemorrhagic brain injury. Exp. Neurol. 206: 43-52.

Ruan ZF, Xu GY and Wu G (2008). Effect of intracerebral injection of thrombin on microglial activation and inducible nitric oxide synthase expression in rats. Chin. J. Cerebrovasc. Dis. 5: 555-559.

Sharp F, Liu DZ, Zhan X and Ander BP (2008). Intracerebral hemorrhage injury mechanisms: glutamate neurotoxicity, thrombin, and Src. Acta Neurochir. Suppl. 105: 43-46.

Ten Cate H (2012). Thrombin generation in clinical conditions. Thromb. Res. 129: 367-370.

Wu H, Zhang Z, Li Y, Zhao R, et al. (2010). Time course of upregulation of inflammatory mediators in the hemorrhagic brain in rats: correlation with brain edema. Neurochem. Int. 57: 248-253.

Xi G, Keep RF and Hoff JT (2006). Mechanisms of brain injury after intracerebral haemorrhage. Lancet Neurol. 5: 53-63.

Xue M and Del Bigio MR (2000). Intracerebral injection of autologous whole blood in rats: time course of inflammation and cell death. Neurosci. Lett. 283: 230-232.

Xue M and Del Bigio MR (2001). Acute tissue damage after injections of thrombin and plasmin into rat striatum. Stroke 32: $2164-2169$ 